\title{
Incidence of tuberculosis among young children in rural Mozambique
}

Elisa López-Varela MD, MPH 1,2, Orvalho Joaquim Augusto MD 1, Kizito Gondo MD 1, Alberto L. García-Basteiro MD MS 1,2, Oscar Fraile MD 1, 2, Tasmiya Ira BS 1, Jose Luis Ribó Aristizabal MD 3, Helder Bulo1, José Muñoz Gutierrez MD PhD 1,2, John Aponte MD PhD 1,2, Eusebio Macete MD PhD 1, Jahit Sacarlal MD PhD 1, Pedro L. Alonso MD PhD 1,2

(1) Manhiça Health Research Centre, Manhiça (CISM). CP 1929 Maputo, Mozambique

(2) Instituto de Salud Global de Barcelona, c/ Rosselló, 132, 5ํㅜa (08036), Barcelona., Spain

(3) Radiology Department, Hospital Sant Joan de Déu Barcelona. Passeig Sant Joan de Déu, 2. 08950 Esplugues de Llobregat, Barcelona, Spain

Running title: Tuberculosis in Mozambique

Abbreviated title: Incidence of Childhood TB in Rural Mozambique

Corresponding author:

Elisa López-Varela, MD, MPH

Centro de Investigação em Saúde de Manhiça (CISM)

CP 1929 Maputo - Moçambique

Tel/Fax (+258) 21810002

Tel. (+258) 21810181 - Cel. (+258) 823168530

Email: elisa.lopez@cresib.cat

Sources of support: This work was supported by the European \& Developing Countries Clinical Trials Partnership [IP_07_32080_003].

Keywords: tuberculosis; pediatrics; epidemiology; incidence; Mozambique 
Background. Tuberculosis contributes significantly to child morbidity and mortality. This study aimed to estimate the minimum community based incidence rate of TB among children $<3$ years of age in Southern Mozambique.

Methods. Between October 2011 and October 2012, in the Manhiça District Health and Demographic Surveillance System (HDSS) we enrolled prospectively all presumptive TB cases younger than under 3 years through passive and active case finding. Participants included all children who were either symptomatic or were close contacts of a notified adult smear positive pulmonary TB. Children were clinically evaluated at baseline and follow-up visits. Investigation for TB disease included chest radiography, HIV and tuberculin skin testing as well as gastric aspirate and induced sputum sampling, which were processed for smear, culture, and mycobacterial molecular identification.

Results. During the study period, 13.764 children <3yr contributed to a total of 9.575 person years. Out of the 789 presumptive TB cases enrolled, 13 had TB culture confirmation and 32 were probable TB cases. The minimum community-based incidence rate of TB (confirmed plus probable cases) was 470/100.000 person-years (95\% Cl: 343 to 629/100.000). HIV co- infection was present in $44 \%$ of the TB cases.

Conclusion. These data highlight the huge burden of pediatric TB. This study provides one of the first prospective population-based incidence data of childhood tuberculosis and adds valuable information to the global effort of producing better estimates, a critical step to inform public health policy. 


\section{Introduction}

Tuberculosis (TB) is an under-recognized but potentially important cause of morbidity and mortality in children in TB endemic settings $s^{1,2}$. Infants and young children (<3years) and those with immunodeficiency caused by HIV or severe malnutrition are at highest risk of developing TB disease following infection ${ }^{3}$. Delay of diagnosis and treatment in these children increases the risk of rapid disease progression and mortality ${ }^{4}$ TB diagnosis is particularly challenging in this population, given the lack of specific symptoms, the difficulty in obtaining samples for microbiological examination, and the often pauci-bacillary disease. The diagnostic yield of samples is often $<20 \%$ under TB program conditions ${ }^{5,6}$. These diagnostic difficulties result in delayed and under-diagnosis of the disease, contributing to the hidden burden of TB in children.

Child TB is receiving more attention ${ }^{3}$ as the World Health Organization (WHO) post2015 TB strategy seeks to engage the wider health sector including the child healthcare sector $^{7}$. The WHO Global Tuberculosis Report 2014 estimates that 550000 children developed tuberculosis during 2013 , representing $6 \%$ of the global TB burden ${ }^{8}$. However, several factors suggest that the true burden of disease may be higher as these estimates assume an equal ratio of notified cases in children and adults (whereas under-reporting in children is very common ${ }^{9}$ ), and estimated deaths only include those in HIV negative children ${ }^{3}$. As a setting's total TB burden increases, there tends to be a rise in the proportion of TB cases attributable to children ${ }^{10}$. Thus, in high TB burden settings, children may represent up to $10-20 \%$ of TB cases, with increased TB incidence in $<5 y$ years ( $y r$ ) and $>15 y r^{4,8,11}$. 
Mozambique is one of the high TB burden countries listed by the WHO but has a very low reported case-detection rate of $37 \%^{8}$. Improved reliable estimates are required to quantify the hidden burden of disease and measure future progress towards the control of TB in the country, especially for vulnerable populations such as children $8,12,13$. We therefore aimed to determine the minimum community-based incidence rate (IR) of childhood TB.

\section{Materials and Methods}

\section{Setting}

The study was conducted in the Manhiça District (rural southern Mozambique), where the Manhiça Health Research Center (Centro de Investigação em Saúde de Manhiça, $\mathrm{CISM}$ ) runs a Health and Demographic Surveillance System (HDSS) including the Manhiça District Hospital (MDH) and other peripheral health posts in the area. The HDSS links demographic and clinical data and covers a population of around 92,000 inhabitants, of which approximately $11 \%$ are $<3 y r^{14}$. A full description of the site can be found elsewhere ${ }^{14}$. In 2011 , the $<5 y$ r mortality rate was $70 / 1000$ live births. Severe malnutrition is common with an estimated IR of 35/1000 person-years among children from $1-2 \mathrm{yr}^{15}$

TB treatment is offered free of charge at the health units and children are routinely vaccinated at birth with Bacille Calmette-Guérin (BCG), with estimated coverage ranging from $86-90 \%{ }^{16,17}$. The 2013 WHO TB incidence estimates for the country is $552 / 100.000$ population ${ }^{8}$. The HIV prevalence in the district is among the highest in the 
world, reaching $39.9 \%$ in the community among individuals aged $18-47 \mathrm{yr}$ and $29.4 \%$ for women attending the antenatal clinic $^{18}$.

\section{Study Design and participants}

A prospective study was designed to recruit participants through passive and active case finding in the community, $\mathrm{MDH}$ and peripheral health centers during a $1 \mathrm{yr}$ period (2011-2012). Participants included all children from the HDSS who were $<3 y r$ at the time of enrolment and had either TB symptoms or were close contacts of a notified adult smear positive pulmonary TB case (PTB). Relapse or recurrent cases were excluded.

\section{Clinical Procedures}

Presumptive TB cases were identified through two strategies: A) Passive case detection of children presenting to the health facility with $\geq 1$ symptoms compatible with TB (see Table 1 for a complete list of symptoms). Those not recruited at the time of the visit to the clinic were later identified through the clinical data collected at the health unit by the HDSS. B) Active case finding consisted of linking the adult smear positive PTB cases registered at the district National TB Program (NTP) in the previous 24 months to the HDSS database in order to identify all household contacts $<3 y r$. At enrolment, demographic and clinical information was collected through interviewing of parents and physical examination. Participants had a chest radiography (CXR) performed, followed by HIV antibody testing and tuberculin skin testing (TST). CXR were performed with a digital X-ray machine and included posteroanterior and lateral projections. For clinical purposes, an initial reading was performed on site by the 
clinician. Subsequently, all CXR were reviewed and re-assessed by an experienced pediatric radiologist (JR) who was blinded to the clinical information. TST was performed with intradermal injection of 2 units (Serum Staten's Institute, Denmark) and reading at 48-96h, according to the study protocol. For symptomatic cases, in the same day 2 ambulatory samples were obtained in a negative pressure facility available at the MDH: one gastric aspirate (GA) and one induced sputum (IS) with nasopharyngeal suction, following WHO recommendations ${ }^{19}$. Asymptomatic cases with abnormal CXR did not undergo sampling but were re-evaluated at further visits. For suspected extrapulmonary TB (EPTB) appropriate samples were obtained.

All case management was performed by the NTP according to established national clinical guidelines. Those patients with clinical or microbiological diagnosis of TB were started on TB treatment at the NTP with the standard 3 or 4 first-line regimens according to WHO category. Other symptomatic patients were referred for specific treatment and follow-up including antibiotics or nutritional supplementation if indicated. Presumptive cases had a follow-up visit within the next six months regardless of initial disease classification to assess resolution of symptoms without anti-TB treatment and /or clinical response to alternative therapy (if any). If persistently symptomatic, further evaluation and testing including CXR and samples was performed to rule out TB. Contacts had a follow-up visit which included physical examination and CXR, as well as GA and IS samples for those symptomatic or with an abnormal CXR.

\section{Laboratory Procedures}


Samples were transported within 4 hours of collection and processed in the Biosafety Level III TB laboratory at CISM. Following $\mathrm{NaCl} / \mathrm{NaOH}$ digestion and concentration through centrifugation, all samples were processed for acid fast bacilli smear testing using LED Microscopy and Zielh Nielsen staining and inoculated into liquid culture media (BACTEC MGIT 960 ${ }^{\circledR}$-automated) and solid media (Lowenstein Jensen). Positive cultures were confirmed using Zielh Nielsen staining and rapid test as well as Xpert MTB/RIF and identified through mycobacterial molecular identification (HAIN GenoType ${ }^{\circledR}$ Mycobacterium CM/AS). First line drug sensitivity testing was performed either on liquid culture or line probe assays. The laboratory is subject to an external quality assurance program.

\section{BOX: Study definitions}

- Exposure to TB was defined as either documented (identified through active case finding) or reported contact (household or regular contact during child lifetime).

- Positive TST was defined as an induration $>5 \mathrm{~mm}$ for HIV or malnourished children and $>10 \mathrm{~mm}$ for the rest of participants.

- HIV infection was defined as: positive antibody test in children $>18$ months (Determine, Abbott Laboratories and confirmed with Unigold, Trinity Biotech); or positive HIV PCR in those $<18$ months; or a strong clinical suspicion with positive antibody test in the absence of a PCR result.

- CXR were classified as compatible if presented $\geq 1$ of the following radiographic abnormalities: airway compression, lymphadenopathy, opacification, nodular picture, effusion, cavities, spondylitis or Ghon focus ${ }^{20}$. 
- Presumptive TB cases included all children $<3$ with compatible TB sign or symptoms.

- Confirmed TB cases included those with compatible symptoms plus a positive culture with Mycobacterium Tuberculosis . Probable TB cases were defined as those with:(1) compatible symptoms unresolved at last clinical follow up visit (prior to any TB treatment initiation) plus ( 2 ) compatible CXR (for children with $\geq 1 C X R$, the latter was used given the likelihood of seeing resolving pneumonias) plus (3) at least one of the following: TB exposure, positive TST or positive response to TB treatment. EPTB cases followed the same definition except for the requirement of having an abnormal CXR. The study TB case definition was adapted a standardized clinical case definition of intrathoracic TB disease and included confirmed plus probable cases $^{21}$ (See Figure 2 for complete case definition).

\section{Ethical approval}

The study protocol was approved by the Mozambican National Bioethics Committee and the Hospital Clinic of Barcelona Ethics Review Committee.

\section{Data Analysis and Statistical Considerations}

Clinical data was double entered in an electronic data capture system (OpenClinica ${ }^{\mathrm{TM}}$ www.openclinica.org) and checked for discrepancies. Statistical software for analysis was Stata 13.0 (StataCorp. 2013. Stata: Release 13. Statistical Software. College Station, TX: StataCorp LP). We calculated z-scores for weight-for-age, height-for-age and weight-for-height using World Health Organization 2006 reference data $^{22}$. 
The minimum community-based IR was calculated as a density rate with the agespecific yearly number of TB cases (according to the study case definition) among study participants divided by the total age-specific population at risk during a period of 12 months (person-time at risk). Time at risk was individually measured using DSS data taking into account demographic events (births, deaths, migrations) of all children included in the study. The IR is considered to be minimum as the case detection system cannot ensure that all TB cases are detected. For each IR 95\% exact Poisson confidence interval were calculated. Proportions were compared using the Pearson or Fisher exact Chi-square test and odds ratio $(\mathrm{OR})$ and $95 \%$ confidence intervals $(\mathrm{Cl})$ were estimated using logistic regression. Variables at a significance level below 0.2 were chosen and placed on stepwise backward multivariate logistic regression. Only factors with a p-value on likelihood ratio tested were retained on the model.

\section{Results}

During the study period, 13.764 children $<3 y r$ contributed to a total of $9.575 p y$ in the Manhiça DSS (Figure1). A total of 789 presumptive TB cases were enrolled (42 and 747 identified through active and passive case finding respectively). Forty-five children fulfilled the TB case definition -13 microbiologically confirmed plus 32 probable TB (Figure 2). Thus, the minimum community-based IR was 470/100.000person-year (95\%Cl 343/100.000 to $629 / 100.000)$ for confirmed plus probable cases and 135/100.000person year for confirmed cases (95\%Cl 72 to 232/100.000)(Table 2).

Baseline characteristics of presumptive TB cases are presented in Table 1. Fifty-four percent were males, and the age distribution showed a predominance of children 
between the ages 12 to 24 months (51\%). The most frequent clinical feature at enrolment was severe malnutrition, which was the only symptom in $72 \%$ of cases. Nutritional assessment found that almost a quarter had severe undernutrition (weight for age $Z$ score $<3$ ). Of the 1347 total CXR performed during the study, $27 \%$ had only one projection. Twenty-one percent of all presumptive TB cases had a CXR compatible with TB. Thirty percent of presumptive cases had a second TST of which, $9 \%$ had a positive TST. Among all presumptive TB cases, nine had a positive smear, although none of the 9 had a positive MTBC culture (4 were NTM and 5 were culture negative). Non-TB mycobacteria (NTM) were isolated in $27 \%$ of all cultures of presumptive cases. We found 7 EPTB cases - 4 lymph node and 3 disseminated- and no TB meningeal cases. A total of 104 children were diagnosed as HIV positive (13\%).

We identified 13 confirmed TB cases ( 7 in GA, 4 in IS and 2 both in GA and IS). The \% of confirmed cases among TB cases was highest for those $<1$ y (40\% vs 29 and $22 \%$ among children with 1-2yr and 2-3yr respectively), and statistically significantly lower for HIV-TB co-infected cases (10 vs $44 \%, p=0.02$ ). Confirmed cases presented a higher frequency of cough or fever as compared to probable cases. Furthermore, the confirmed cases appeared to be more symptomatic at enrolment than did the probable cases ( $53.8 \%$ vs $15.6 \%$ presenting with $\geq 1$ TB symptom respectively, $p<0.001)$. Probable cases had a higher proportion of HIV-infection $(p=0.01)$, positive TST $(p=0.001)$ or BCG scarring $(p=0.08)$ as compared to confirmed cases.

Multivariate logistic regression analysis for TB risk factors showed that HIV infection, and number of previous outpatient consultations were predictors of TB disease when 
compared to unlikely TB cases. After adjusting for other variables, HIV infected children were six times more likely to have TB disease than uninfected ones (OR 8.4, 95\% $\mathrm{Cl} 4$ to 17) (Table 3, available online).

Fifty-two patients were started on TB treatment based on clinical or microbiological criteria, $67 \%$ fulfilled the study TB case definition (Table 4). A total of 97 children initiated isoniazide preventive treatment (IPT) (71 based on exposure history, 21 on TST results and 5 unspecified) and 5 were later diagnosed as TB cases while on IPT. Due to drug supply shortages, isoniazide was not always available and $47 \%$ of children with criteria did not initiate IPT. The mortality rate for all presumptive cases at 12 months after enrolment was $5.2 \%$ and increased with decreasing age $(10.9,5.7$ and $0.8 \%$ of children in the first, second and third year of life respectively, $p<0.001)$. Mortality was also higher in TB cases as compared to non-TB ( $13 \%$ vs $5 \%$ respectively, $p=0.02)$ as it was in HIV infected children as compared to HIV-uninfected $(14.4 \%$ vs $3.8 \%, p<0.001)$. The case fatality rate was $9 \%$ ( $N=4 / 45$ TB cases), all deaths taking place in the first 6 months after enrollment.

\section{Discussion}

This study provides one of the first prospective population-based incidence estimates of childhood tuberculosis in a high TB-HIV endemic setting and shows a consistently high IR across all ages. These results underscore the hypothesis of a gross underdetection and under-reporting of childhood TB in Mozambique and globally ${ }^{23}$. 
Mozambique has almost half its population below the age of $<15 \mathrm{yr}$, and yet, pediatric TB only accounted for 7\% of all new cases notified in 2012, much lower than the expected $10-20 \%$ of the total burden of TB disease seen in high burden countries. In the Manhiça District, the notified IR for children <1yr and between 1-4 yr in 2011 was 163 and 399/100.000 respectively (personal communication ${ }^{24}$ ). This corresponds to half the TB IR reported in this study and may suggest under-detection and underdiagnosing. Furthermore, under-detection is common in the wider Mozambican context, where WHO estimates that only $37 \%$ of actual TB cases are detected ${ }^{8}$. While the latest WHO country incidence estimates are 552 per 100.000 population, data from Manhiça suggest that the burden of disease in Southern Mozambique might be much higher. In fact, while the national 2011 notified rate was 186/100.000 population, same-year data from the Manhiça suggest the TB incidence rate of smear positive cases could be as high as 456 per 100,000 population among adults aged 18 to $47 \mathrm{yr}^{25}$.

Globally, several pediatric TB incidence estimates have been published recently, with results varying from less than 200000 new cases in $2013^{26}$ to 970000 in $2010^{6}$. The large variation in the estimates highlights the challenges in estimating the burden of pediatric TB and the need for population-based data to inform predictive models.

There are few studies reporting age-specific pediatric TB incidence in high burden countries. Most studies are based on hospital-based retrospective reviews of notification rates and, to our knowledge, none have reported community IR using DSS ${ }^{11,27-35}$. However, in areas where health seeking behavior strongly modifies the pattern 
of attendance, community based studies that use active case detection rather than notified TB rates are necessary to provide accurate estimates. Moreover, childhood mortality and frequent migration are potential causes of disease underestimation if DSS person-years are not available. Inconsistency in TB clinical definitions among studies are a challenge for comparability and have been a limitation in obtaining data for meta-analyses. The recently proposed definition, applied in this study, may pave the road for future comparisons ${ }^{9}$. The IR we report is significantly higher than in other high burden African countries such as Malawi (notified IR<1y of 78/100.000) or Tanzania (theoretical IR $<5 y$ based on likelihood of disease progression of 134.5 to 308.5/100.000) and similar to data from Gabon (extrapolated IR<15y 366/100.000), or neighboring South Africa (notified IR<5y 770/100.000) 303132 .

There are several limitations to this study, mostly leading to a possible underestimation of the true TB incidence. First, only single-day samples were obtained as most patients would not accept overnight admission, decreasing the chances of microbiological confirmation. Second, for study purposes CXR were read by a single blinded experienced pediatric radiologist rather than the two independent CXR readers are often recommended to prevent bias, given the pivotal role of CXR in case definition, and the poor inter and intra-observer agreement among reviewers ${ }^{36}$. Third, contact tracing could not be fully implemented mainly due to difficulties in patient identification and poor recording.. Fourth, the \% of EPTB cases was lower than the 20$30 \%$ expected and reported by others ${ }^{11,30,31,37-39}$. While BCG protection may have a role, it is likely that some EPTB cases in this study were missed; the reason may be due to a stronger focus on pulmonary TB in the study design, errors in classification 
(disease localization, including disseminated TB, may be confounding in young children in the absence of CT-scan), or lost cases due to the fact that severely ill children are often transferred to the tertiary reference hospital in the capital for specific diagnostic procedures. Finally, there is a risk of overestimating TB IR by either including TB prevalent cases at enrollment or adding new incident cases during follow-up beyond the one year enrollment period. Although this possibility can't be ruled out, we believe the effect would be minimal and probably overweighed by the above-mentioned risk of underestimation.

In this study, HIV prevalence was high regardless of disease classification, reaching $56 \%$ of probable cases. The fact that significantly fewer HIV infected children had TB confirmation reflects the diagnostic difficulties in this group. Given the overlap between symptoms from both TB and HIV, HIV infected cases of TB pose the greatest ascertainment bias with the highest risk of over or under estimation. Even though IPT is indicated in all HIV positive children, the implementation of IPT among African NTP remains very poor ${ }^{40}$, and in this study, the high proportion of missed opportunities for chemoprophylaxis in HIV and or TB exposed should raise alert. There may also be missed preventable child deaths in HIV-infected presumptive TB cases and possible TB cases. It has been reported that many children who die of diseases such as malnutrition or respiratory infections may have in fact, undiagnosed $\mathrm{TB}^{41}$. There is thus a need for widespread recognition that TB control is crucial for childhood survival ${ }^{1}$. 
This study highlights the huge burden of pediatric TB under detection in children under

3. These data add valuable information to the global effort of producing better estimates of childhood TB burden, a critical step to inform public health policy.

\section{Acknowledgement}

We thank the participants and their families. We are grateful to the National TB Program for, health staff at the Manhiça District Hospital and peripheral health centers for their contribution. We thank Dr Bassat and Dr Naniche(Barcelona Centre for International Health Research) for reviewing the paper. 


\section{References}

1 Graham SM, Sismanidis C, Menzies HJ, Marais BJ, Detjen AK, Black RE. Importance of tuberculosis control to address child survival. Lancet 2014; 6736: 5-7.

2 Marais BJ, Schaaf HS, Graham SM. Child health and tuberculosis. Lancet Respir Med 2014; 2: 254-6.

3 Organization WH. Roadmap for childhood tuberculosis: towards zero deaths. 2013.http://apps.who.int/iris/handle/10665/89506 (accessed 18 Nov2013).

4

5

6

7

8 Perez-Velez CM, Marais BJ. Tuberculosis in children. N Engl J Med 2012; 367: 348-61. Nicol MP, Zar HJ. New specimens and laboratory diagnostics for childhood pulmonary TB: progress and prospects. Paediatr Respir Rev 2011; 12: 16-21.

Jenkins $\mathrm{HE}$, Tolman AW, Yuen CM, et al. Incidence of multidrug-resistant tuberculosis disease in children: systematic review and global estimates. Lancet 2014; 6736: 1-8. WHO | WHO End TB Strategy. http://www.who.int/tb/post2015_strategy/en/ (accessed 15 Nov2014).

WHO. Global Tuberculosis Report 2014. Geneva, Switzerland: World Health Organization, 2014. http://www.who.int/tb/publications/global_report/en/ (accessed 24 Jun2013).

Marais BJ, Graham SM, Maeurer M, Zumla A. Progress and challenges in childhood tuberculosis. Lancet Infect Dis 2013; 13: 287-9.

Donald PR. Childhood tuberculosis: out of control? Curr Opin Pulm Med 2002; 8: 17882.

Moyo S, Verver $\mathrm{S}$, Mahomed $\mathrm{H}$, et al. Age-related tuberculosis incidence and severity in children under 5 years of age in Cape Town, South Africa. Int J Tuberc Lung Dis 2010; 14: 149-54.

López-Varela E, García-Basteiro AL. World health organization guidelines for childhood tuberculosis management: successes achieved and challenges ahead. Pediatr Infect Dis J 2014; 33: 1310-1.

3 García-Basteiro AL, López-Varela E, Manhiça I, Macete E, Alonso PL. Mozambique faces challenges in the fight against tuberculosis. Lancet 2014; 383: 215-6.

4 Sacoor C, Nhacolo A, Nhalungo D, et al. Profile: Manhica Health Research Centre (Manhica HDSS). Int J Epidemiol 2013; 42: 1309-18.

5 Nhampossa $T$, Sigaúque B, Machevo $S$, et al. Severe malnutrition among children under the age of 5 years admitted to a rural district hospital in southern Mozambique. Public Health Nutr 2013; 16: 1565-74.

6 Consonni D, Montenegro Agorostos Karagianis MM, Bufardeci G. Immunisation with BCG in the Maringue District, Sofala Province, Mozambique. Tuberc Res Treat 2013; 2013: 312065.

17 WHO vaccine-preventable diseases: monitoring system. 2013 Global Summary. Mozambique. Available at:

http://apps.who.int/immunization_monitoring/en/globalsummary. Accessed on January2014.

8 González R, Munguambe K, Aponte J, et al. High HIV prevalence in a southern semirural area of Mozambique: a community-based survey. HIV Med 2012; 13: 581-8.

9 Guidance for national tuberculosis programmes on the management of tuberculosis in children. 2006.

Cuevas LE, Browning R, Bossuyt $\mathrm{P}$, et al. Evaluation of tuberculosis diagnostics in children: 2. Methodological issues for conducting and reporting research evaluations of tuberculosis diagnostics for intrathoracic tuberculosis in children. Consensus from an expert panel. J Infect Dis 2012; 205 Suppl : S209-15. 
21 Graham SM, Ahmed T, Amanullah F, et al. Evaluation of tuberculosis diagnostics in children: 1. Proposed clinical case definitions for classification of intrathoracic tuberculosis disease. Consensus from an expert panel. J Infect Dis 2012; 205 Suppl : S199-208.

22 World Health Organization. WHO childgrowthstandards: length/height-for-age, weightfor-age, weight-for-length, weight-for-height and bodymassindex-for-age: methods and development. Geneva. .

23 Lestari T, Probandari A, Hurtig A-K, Utarini A. High caseload of childhood tuberculosis in hospitals on Java Island, Indonesia: a cross sectional study. BMC Public Health 2011; 11: 784.

24 García-Basteiro, A; López-Varela, E; Joaquim Augusto, O; Aponte, J; Macete, E; Manhiça, I; Alonso P. Determining the minimum tuberculosis incidence risk among adults in the district of Manhiça, Mozambique [abstract PC-441-01]. In: 44th Union World Conference on Lung Health. Paris, France. November 2013. , 2013.

25 García-Basteiro AL, López-Varela E, Respeito D, et al. High tuberculosis burden among people living with HIV in southern Mozambique. Eur Respir J 2014. doi:10.1183/09031936.00145714.

26 Murray CJL, Ortblad KF, Guinovart C, et al. Global, regional, and national incidence and mortality for HIV, tuberculosis, and malaria during 1990-2013: a systematic analysis for the Global Burden of Disease Study 2013. Lancet 2014; 384: 1005-70.

27 Donald PR, Maher D, Qazi S. A research agenda to promote the management of childhood tuberculosis within national tuberculosis programmes. Int J Tuberc Lung Dis 2007; 11: 370-80.

28 Marais BJ, Gie RP, Schaaf HS, et al. The natural history of childhood intra-thoracic tuberculosis: a critical review of literature from the pre-chemotherapy era. Int. J. Tuberc. Lung Dis. 2004; 8: 392-402.

29 Marais BJ, Hesseling a C, Gie RP, Schaaf HS, Beyers N. The burden of childhood tuberculosis and the accuracy of community-based surveillance data. Int J Tuberc Lung Dis 2006; 10: 259-63.

30 Harries a D, Hargreaves NJ, Graham SM, et al. Childhood tuberculosis in Malawi: nationwide case-finding and treatment outcomes. Int J Tuberc Lung Dis 2002; 6: 42431.

31 Praygod G, Todd J, McDermid JM. Early childhood tuberculosis in northwestern Tanzania. Int J Tuberc Lung Dis 2012; 16: 1455-60.

32 Van Rie a, Beyers N, Gie RP, Kunneke M, Zietsman L, Donald PR. Childhood tuberculosis in an urban population in South Africa: burden and risk factor. Arch Dis Child 1999; 80: 433-7.

33 Mahomed $\mathrm{H}$, Ehrlich R, Hawkridge $\mathrm{T}$, et al. TB incidence in an adolescent cohort in South Africa. PLoS One 2013; 8: e59652.

34 Nantongo JM, Wobudeya $\mathrm{E}$, Mupere $\mathrm{E}$, et al. High incidence of pulmonary tuberculosis in children admitted with severe pneumonia in Uganda. BMC Pediatr 2013; 13: 16.

35 Ssengooba W, Colebunders R. Burden of tuberculosis disease among adolescents in a rural cohort in Eastern Uganda. 2013. doi:10.1186/1471-2334-13-349.

36 Swingler GH, du Toit G, Andronikou S, van der Merwe L, Zar HJ. Diagnostic accuracy of chest radiography in detecting mediastinal lymphadenopathy in suspected pulmonary tuberculosis. Arch Dis Child 2005; 90: 1153-6.

37 Cavanaugh J, Genga K, Marigu I, Laserson K, Ackers M, Cain K. Tuberculosis among children in Kenya: epidemiology and impact of HIV in two provinces. J Trop Pediatr 2012; 58: 292-6.

38 Kapata N, Chanda-Kapata P, O'Grady J, et al. Trends in childhood tuberculosis in Zambia: a situation analysis. J Trop Pediatr 2013; 59: 134-9. 
39 Flamen a, Bélard S, Kokou C, Janssen S, Grobusch MP. Childhood tuberculosis in Lambaréné, Gabon: tuberculosis control in its infancy? Infection 2013. doi:10.1007/s15010-013-0529-5.

40 Schaaf HS, Marais BJ. Management of multidrug-resistant tuberculosis in children: a survival guide for paediatricians. Paediatr Respir Rev 2011; 12: 31-8.

41 Chintu C, Mudenda V, Lucas S, et al. Lung diseases at necropsy in African children dying from respiratory illnesses: a descriptive necropsy study. Lancet 2002; 360: 985-90. 
Table 1. Baseline characteristics of presumptive TB cases $(n=789), N(\%)$

\begin{tabular}{|c|c|}
\hline Sex (male) & $430(54,5)$ \\
\hline Age in months (Median [IQR]) & $19.8(13.8-25.9)$ \\
\hline \multicolumn{2}{|l|}{ Age group } \\
\hline$<1 \mathrm{yr}$ & $146(18.5)$ \\
\hline $1-2 \mathrm{yr}$ & $403(51.1)$ \\
\hline$>2 \mathrm{yr}$ & $240(30.4)$ \\
\hline BCG scar $(n=785)$ & $686(87.4)$ \\
\hline$>1$ hospitalization in previous year & $206(26.1)$ \\
\hline$\geq 10$ consultations in previous year & $131(16.6)$ \\
\hline TB contact (documented or reported) & $87(11)$ \\
\hline \multicolumn{2}{|l|}{ Symptoms * } \\
\hline Cough $\geq 2$ weeks & $156(19.8)$ \\
\hline Fever $\geq 2$ weeks & $50(6.3)$ \\
\hline Malnutrition (chronic or acute) & $668(84.7)$ \\
\hline Wheeze or lower respiratory infection & $43(5.5)$ \\
\hline Adenopathy & $3(0.4)$ \\
\hline \multicolumn{2}{|l|}{ Number of presenting symptoms } \\
\hline One symptom & $608(77.1)$ \\
\hline Only malnutrition & $565(71.6)$ \\
\hline Hospitalized at time of enrolment & $101(12.8)$ \\
\hline \multicolumn{2}{|l|}{ Physical examination } \\
\hline Stunting $(n=775)+$ & $404(52.1)$ \\
\hline Undernutrition $(\mathrm{n}=777) \ddagger$ & $184(23.7)$ \\
\hline Wasting $(n=775) ¥$ & $92(11.9)$ \\
\hline Kwashiorkor $(n=780)$ & $33(4.2)$ \\
\hline Febrile $(\mathrm{N}=780)$ & $44(5.6)$ \\
\hline Crackles on chest examination & $27(3.4)$ \\
\hline TST positive ( $n=787)$ & $74(9.4)$ \\
\hline HIV positive & $104(13.2)$ \\
\hline Radiological changes suggestive of TB $(n=766)$ & $160(20.9)$ \\
\hline
\end{tabular}

\section{Footnote:}

Abbreviations: IQR, interquartile range; BCG, Bacille Calmette Guerin; HIV, human immunodeficiency virus; TST, tuberculin skin test

* Compatible TB symptoms: cough for $\geq 14$ days not responding to appropriate course of antibiotics; fever greater than $38^{\circ} \mathrm{C} \geq 14$ days . after common causes like malaria or pneumonia were excluded; malnutrition defined as under $60 \%$ weight for height, failure to gain weight for more than 2 months or any loss of weight and not responded to nutritional interventional; unexplained wheeze $\geq 14$ days not responding to standard treatments; lower respiratory tract infection $\geq 14$ days not responding to antibiotics after 72 hours; TB exposure in the last 12 months; symptoms compatible with extrapulmonary TB (EPTB) such as painless enlarged lymph nodes with or without fistula formation $\geq 14$ days, arthritis, gibbus, meningitis, effusion, or unexplained hematuria, dysuria or polaquiuria for $\geq 21$ days.

$\dagger$ Stunting: Height for age $\mathrm{Z}$ score $<2$.

$\neq$ Undernutrition: Weight for age $\mathrm{Z}$ score $<2$.

$¥$ Wasting: Weight for height $<2$. 
Table 2: Community based incidence rate of confirmed and probable tuberculosis cases by age group

All tuberculosis cases

\begin{tabular}{lccccc}
\hline & Cases & PY & $\begin{array}{c}\text { Incidence Rate } \\
\text { per 100000py }\end{array}$ & $\begin{array}{c}\text { 95\% Confidence } \\
\text { Interval }\end{array}$ \\
\hline Confimed & 13 & 9575,6 & 135,8 & 72,3 & 232,2 \\
Probable & 32 & 9575,6 & 334,2 & 228,6 & 471,8 \\
Total & 45 & 9575,6 & 470,0 & 342,8 & 628,8 \\
\hline
\end{tabular}

Confirmed cases by age group

\begin{tabular}{|c|c|c|c|c|c|}
\hline \multirow{2}{*}{$\frac{\text { Age (years) }}{<1}$} & \multirow{2}{*}{$\begin{array}{c}\text { Cases } \\
4\end{array}$} & \multirow{2}{*}{$\begin{array}{c}\text { PY } \\
3265,6\end{array}$} & \multirow{2}{*}{$\begin{array}{c}\text { Incidence Rate } \\
\text { per 100000py }\end{array}$} & \multicolumn{2}{|c|}{$\begin{array}{l}\text { 95\% Confidence } \\
\text { Interval }\end{array}$} \\
\hline & & & & 33,3 & 313,3 \\
\hline $1-2$ & 5 & 3199,4 & 156,3 & 68,9 & 407,8 \\
\hline $2-+$ & 4 & 3110,5 & 128,6 & 52,2 & 374,5 \\
\hline All Confirmed & 13 & 9575,6 & 135,8 & 72,3 & 232,2 \\
\hline
\end{tabular}

Probable cases by age group

\begin{tabular}{cccccc}
\hline Age (years) & Cases & PY & $\begin{array}{c}\text { Incidence Rate } \\
\text { per 100000py }\end{array}$ & \multicolumn{2}{c}{$\begin{array}{c}\text { 95\% Confidence } \\
\text { Interval }\end{array}$} \\
\hline$<1$ & 6 & 3265,6 & 183,7 & 67,4 & 399,9 \\
$1-2$ & 12 & 3199,4 & 375,1 & 193,8 & 655,2 \\
$2-+$ & 14 & 3110,5 & 450,1 & 246,1 & 755,2 \\
All Probable & 32 & 9575,6 & 334,2 & 228,6 & 471,8 \\
\hline
\end{tabular}

All cases by age group

\begin{tabular}{cccccc}
\hline Age & Cases & PY & $\begin{array}{c}\text { Incidence Rate } \\
\text { per 100000py }\end{array}$ & \multicolumn{2}{c}{$\begin{array}{c}\text { 95\% Confidence } \\
\text { Interval }\end{array}$} \\
\hline$<1$ & 10 & 3265,6 & 306,2 & 146,9 & 563,2 \\
$1-2$ & 17 & 3199,4 & 531,4 & 309,5 & 850,7 \\
$2-+$ & 18 & 3110,5 & 578,7 & 343,0 & 914,6 \\
All TB Cases & 45 & 9575,6 & 470,0 & 342,8 & 628,8 \\
\hline
\end{tabular}

Footnote:

Abbreviations: PY, person years 
Table 3. TB risk factor analysis

\begin{tabular}{|c|c|c|c|c|c|c|c|c|}
\hline & \multirow{2}{*}{\multicolumn{2}{|c|}{ TB Unlikey $(\mathrm{N}=603)$}} & \multirow{2}{*}{\multicolumn{2}{|c|}{ TB Case $(\mathrm{N}=45)$}} & \multicolumn{2}{|c|}{ Univariate } & \multicolumn{2}{|c|}{ Adjusted OR (95\% CI) } \\
\hline & & & & & OR $(95 \% \mathrm{Cl})$ & $\mathbf{p}$ & OR $(95 \% \mathrm{Cl})$ & $\mathbf{p}$ \\
\hline \multicolumn{9}{|l|}{ Sex } \\
\hline Male & 322 & $53,4 \%$ & 20 & $44,4 \%$ & - & & & \\
\hline Female & 281 & $46.6 \%$ & 25 & $55,6 \%$ & $1.43(0.78-2.64)$ & 0.246 & $1.52(0.79-2.92)$ & 0.210 \\
\hline $\begin{array}{l}\text { Age in months (Median } \\
[\mathrm{IQR}])\end{array}$ & 19.8 & $.1-26.0)$ & 19.5 & $3.8-26.4)$ & & & & \\
\hline \multicolumn{9}{|l|}{ Age category, $\mathrm{N}(\%)$} \\
\hline$<1$ & 100 & $16,6 \%$ & 10 & $22,2 \%$ & - & & & \\
\hline $1-2$ & 318 & $52,7 \%$ & 17 & $37,8 \%$ & $0.53(0.24-1.21)$ & & $0.51(0.21-1.23)$ & 0.133 \\
\hline $2-+$ & 185 & $30,7 \%$ & 18 & $40,0 \%$ & $0.95(0.43-2.19)$ & 0.153 & $0.94(0.39-2.26)$ & 0.890 \\
\hline \multicolumn{9}{|l|}{ BCG Scar } \\
\hline Absent & 74 & $12,3 \%$ & 7 & $15,6 \%$ & - & & & \\
\hline Present & 527 & $87,7 \%$ & 37 & $82,2 \%$ & $0.74(0.32-1.73)$ & 0.488 & & \\
\hline \multicolumn{9}{|l|}{ TB contact } \\
\hline No & 557 & $92,4 \%$ & 32 & $71,1 \%$ & - & & & \\
\hline Yes & 46 & $7,6 \%$ & 13 & $28,9 \%$ & $4.92(2.38-10.15)$ & $<0.001$ & & \\
\hline \multicolumn{9}{|l|}{$\begin{array}{l}\text { № consultations in } \\
\text { previous year }\end{array}$} \\
\hline Median (IQR) & \multicolumn{2}{|c|}{$5(3-8)$} & \multicolumn{2}{|c|}{$7(2-11)$} & - & 0.074 & & \\
\hline$<10$ & 505 & $83,7 \%$ & 29 & $64,4 \%$ & - & & & \\
\hline $10-+$ & 98 & $16,3 \%$ & 16 & $35,6 \%$ & $2.84(1.48-5.47)$ & 0.001 & $2.72(1.35-5.49)$ & 0.005 \\
\hline \multicolumn{9}{|l|}{$\begin{array}{l}\text { № of hospitalizations in } \\
\text { previous year }\end{array}$} \\
\hline None & 446 & $74,0 \%$ & 26 & $57,8 \%$ & - & & & \\
\hline At least one & 157 & $26,0 \%$ & 19 & $42,2 \%$ & $2.08(1.11-3.87)$ & 0.019 & & \\
\hline \multicolumn{9}{|l|}{ Symptoms at enrollment } \\
\hline \multicolumn{9}{|l|}{ Cough $\geq 2$ weeks } \\
\hline no & 489 & $81,1 \%$ & 36 & $80,0 \%$ & - & & & \\
\hline yes & 114 & $18,9 \%$ & 9 & $20,0 \%$ & $1.07(0.50-2.29)$ & 0.857 & & \\
\hline \multicolumn{9}{|l|}{ Fever $\geq 2$ weeks } \\
\hline no & 567 & $94,0 \%$ & 40 & $88,9 \%$ & - & & & \\
\hline yes & 36 & $6,0 \%$ & 5 & $11,1 \%$ & $1.97(0.73-5.30)$ & 0.172 & & \\
\hline \multicolumn{9}{|c|}{$\begin{array}{l}\text { Chronic or Acute } \\
\text { Malnutrition malnutrition }\end{array}$} \\
\hline no & 82 & $13,6 \%$ & 12 & $26,7 \%$ & - & & & \\
\hline yes & 521 & $86,4 \%$ & 33 & $73,3 \%$ & $0.43(0.21-0.88)$ & 0.016 & $0.42(0.19-0.89)$ & 0.024 \\
\hline \multicolumn{9}{|l|}{ Physical Exam } \\
\hline \multicolumn{9}{|l|}{ Stunting † } \\
\hline no & 281 & $46,6 \%$ & 16 & $35,6 \%$ & - & & & \\
\hline yes & 312 & $51,7 \%$ & 28 & $62,2 \%$ & $1.58(0.83-2.98)$ & 0.158 & & \\
\hline \multicolumn{9}{|l|}{ Undernutrition $\ddagger$} \\
\hline no & 460 & $76,3 \%$ & 30 & $66,7 \%$ & - & & & \\
\hline yes & 134 & $22,2 \%$ & 14 & $31,1 \%$ & $1.60(0.82-3.11)$ & 0.161 & & \\
\hline \multicolumn{9}{|l|}{ Wasting $¥$} \\
\hline no & 531 & $88,1 \%$ & 37 & $82,2 \%$ & - & & & \\
\hline yes & 62 & $10,3 \%$ & 7 & $15,6 \%$ & $1.62(0.69-3.80)$ & 0.262 & & \\
\hline
\end{tabular}


Crackles on chest

examination

no

yes

$584 \quad 97,0 \% \quad 39 \quad 90,7 \%$

Tuberculin skin test

Postive

$18 \quad 3,0 \% \quad 4 \quad 9,3 \%$

$3.32(1.07-10.36) \quad 0.052^{*}$

Negative

$0 \quad 0,0 \% \quad 23 \quad 51,1 \%$

HIV Reported

Not positive

$603 \quad 100,0 \% \quad 21$

$46,7 \%$

$<0.001 \mu$

Postive

$549 \quad 91,0 \% \quad 25 \quad 55,6 \%$

$54 \quad 9,0 \% \quad 20 \quad 44,4 \%$

$8.13(4.12-16.04)<0.001$

$8.42(4.26-16.62)<0.001$

\section{Footnote:}

Abbreviations: IQR, interquartile range; BCG, Bacille Calmette Guerin; HIV, human immunodeficiency virus; TST, tuberculin skin test

† Stunting: Height for age Z score $<2$.

$\neq$ Undernutrition: Weight for age $\mathrm{Z}$ score $<2$.

$¥$ Wasting: Weight for height $<2$

$\mu$ This variable was not included in multivariate analysis as a positive TST is part of the TB definition

* Exact Chi-squared test 
Table 4. Outcome by group classification, $N(\%)$

\begin{tabular}{|c|c|c|c|c|c|c|}
\hline & $\begin{array}{l}\text { Presumptive } \\
(\mathrm{N}=789)\end{array}$ & $\begin{array}{l}\text { Definite } \\
(\mathrm{N}=13)\end{array}$ & $\begin{array}{c}\text { Probable } \\
(\mathrm{N}=32)\end{array}$ & $\begin{array}{c}\text { Possible } \\
(\mathrm{N}=96)\end{array}$ & $\begin{array}{l}\text { Unlikely } \\
(\mathrm{N}=603)\end{array}$ & $\begin{array}{l}\text { MTB infection } \\
(\mathrm{N}=45)\end{array}$ \\
\hline Sex (male) & $430(54.5 \%)$ & $5(38.5 \%)$ & $15(46.9 \%)$ & $57(59.4)$ & $322(53.4 \%)$ & $31(68.9 \%)$ \\
\hline HIV positive & $104(13.2 \%)$ & $2(15.4 \%)$ & $18(56.3 \%)$ & $24(25 \%)$ & $54(9 \%)$ & $6(13.3 \%)$ \\
\hline IPT & $64(8.1 \%)$ & $1(7.7 \%)$ & $4(12.5 \%)$ & $4(4.2 \%)$ & $24(4 \%)$ & $31(68.9 \%)$ \\
\hline TB treatment & $52(6.6 \%)$ & 9 (69.2\%) & $26(81.3 \%)$ & 14 (14.6\%) & $1(0.2 \%)$ & $2(4.4 \%)$ \\
\hline $\begin{array}{l}\text { Median time to diagnosis } \\
\text { (days) N (IQR) }\end{array}$ & $115(35-224)$ & $41(35-115)$ & $184(55-224)$ & $60(35-235)$ & $14(14-14)$ & $148(89-207)$ \\
\hline Mortality at 12 months & $41(5.2)$ & $3(23.1 \%)$ & $1(3.1 \%)$ & $13(13.5 \%)$ & $23(3.8 \%)$ & $1(2.2 \%)$ \\
\hline
\end{tabular}

\section{Footnote:}

Abbreviations: HIV, human immunodeficiency virus; IPT, isoniazide preventive treatment; IQR, interquartile range; TST, tuberculin skin test 
Figure 1. Study Profile

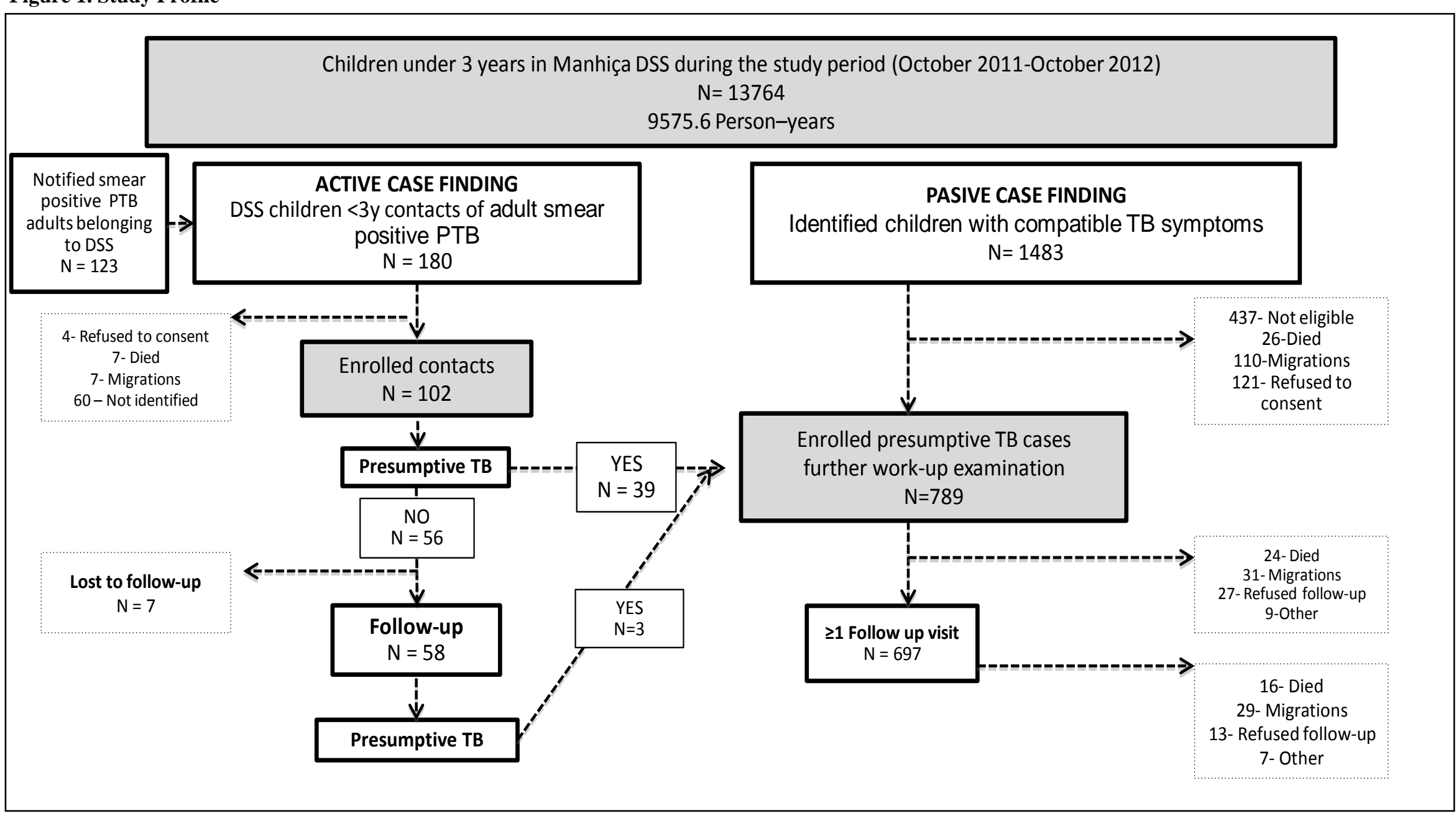


Figure 2. Algorithm for study case classification adapted from Graham et al (JID, 2012).

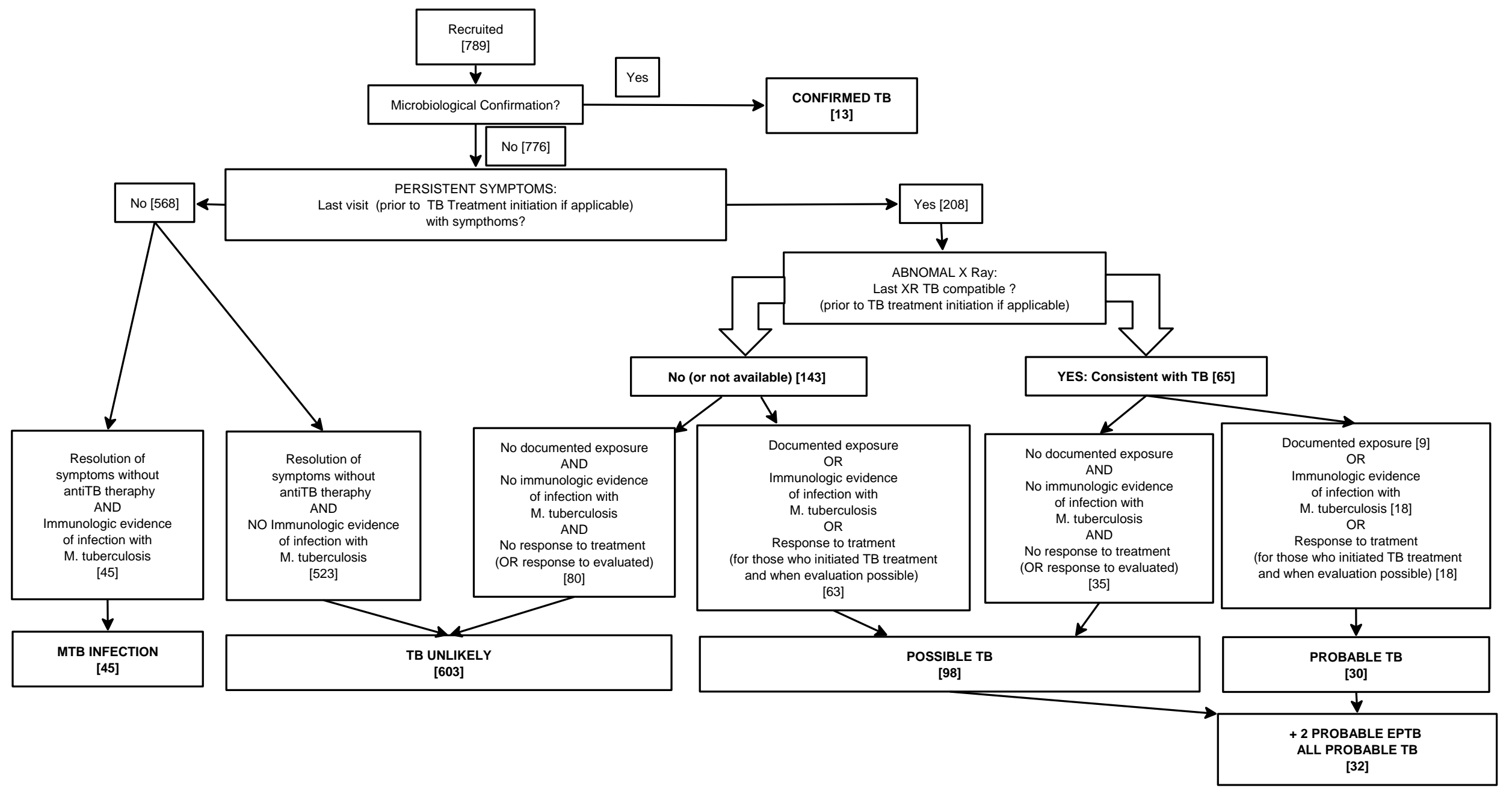




\section{Figure 1. Study Profile}

Flowchart showing the number of children under three in the study area and those enrolled in the study. A total of 1483 children were identified with at least one compatible TB symptom and 747 presumptive TB cases were enrolled in the study for further workup. Among the 329 adult smear positive cases registered at the NTP between October 2010-October 2012, we identified 123 belonging to the study area and 180 contacts $<3$, of whom 102 accepted to participate in the study yielding an additional 42 presumptive TB cases. Among the remaining 60 contacts, 7 were lost to follow-up and the rest had at least one follow up visit. Eighty-eight percent presumptive TB cases enrolled had at least one follow-up visit and 632/697 completed follow-up (had follow-up visits until alternative diagnosis was made or became asymptomatic). Abbreviations: DSS, Demographic Surveillance System; PTB, pulmonary tuberculosis.

\section{Figure 2. Algorithm for study case classification adapted from Graham et al (JID,}

\section{2)}

Definitions. Confirmed TB: Compatible symptoms plus a positive culture with MTBC Persistent symptoms: Compatible symptoms unresolved at last clinical follow up visit (prior to any TB treatment initiation). Consistent CXR: CXR read by blinded experienced pediatric radiologist with one or more radiographic abnormalities (airway compression, lymphadenopathy, opacification, nodular picture, effusion, cavities, spondylitis or Ghon focus). For children with more than one CXR, the latter was used given the likelihood of seeing resolving pneumonias. Positive response to TB treatment: determined by the clinician at first possible follow-up visit and defined as total/partial resolution of clinical features suggestive of TB present at baseline with no new clinical features suggestive of TB for those patients adherent to TB treatment. Resolution of symptoms without anti-TB treatment was evaluated by the clinical at first follow-up visit as total/partial resolution of 
clinical symptoms suggestive of TB present at baseline and no new clinical features suggestive of TB in the absence of TB treatment.

Description: Of the initial 789 presumptive TB cases, 568 had symptoms resolved at follow-up visits, leaving 208 presumptive cases with persistent TB symptoms, of which 65 had an abnormal CXR at last visit. Probable cases include 30 children who fulfilled the definition plus an additional 2 extrapulmonary-cases. Contacts not enrolled as presumptive TB cases are not included in the algorithm. 